ISSN 2447-9071

doi https://doi.org/10.36414/rbmc. v5i13.17

Contato para correspondência: Luana Cristina Barbosa Estrêla Alves

E-mail:

estrela.luana@gmail.com

Conflito de interesse: Não

Financiamento: Recursos próprios

Recebido: 01/11/2019

Aprovado: 13/12/2019

\section{Conhecimento de acadêmicos da saúde sobre a atuação do fisioterapeuta em unidades de terapia intensiva}

\author{
Knowledge of academic of health about the physiotherapist \\ performance in intensive therapy units
}

\author{
Luana Cristina Barbosa Estrêla Alves ${ }^{1}$, Luiza Parreira Thommen ${ }^{1}$, Clayson Moura Gomes ${ }^{1,2}$ \\ Valéria Rodrigues Costa de Oliveira ${ }^{1}$ \\ ${ }^{1}$ Pontifícia Universidade Católica de Goiás - PUC Goiás \\ ${ }^{2}$ Faculdade da Polícia Militar - FPM
}

\section{Resumo}

A Unidade de Terapia Intensiva (UTI) é definida como uma área crítica destinada à internação de pacientes graves, que requerem atenção profissional especializada de forma contínua, materiais específicos e tecnologias necessárias ao diagnóstico, monitorização e terapia. Para prover uma adequada assistência, deve ser designada uma equipe multiprofissional, legalmente habilitada, sendo que atribuições e as responsabilidades de todos os profissionais que atuam na unidade devem estar formalmente designadas, descritas e divulgadas aos profissionais e acadêmicos. Oobjetivo desteestudo foide identificar o nível de conhecimento de estudantes da área da saúde de uma Instituição de Ensino Superior de Goiás acerca do papel do Fisioterapeuta na equipe multiprofissional da UTI. Trata-se de um estudo transversal, descritivo com abordagem quantitativa, com 73 acadêmicos dos cursos de Enfermagem, Medicina e Nutrição. Foi utilizado como instrumento um questionário elaborado pelas pesquisadoras especificamente para o presente estudo. Na análise estatística foi utilizado o software Graphpad Prism versão 5.0 onde foi analisada a significância do questionário aplicado no estudo com o Teste TStudent pareado paramétrico, definiu-se para o estudo um nível de significância de p<0,05. Verificou-se os acadêmicos dos três cursos atribuíram corretamente ao fisioterapeuta mais de $60 \%$ das condutas apresentadas, e que os alunos de Medicina tiveram percentual de acerto significativamente maior em relação aos demais. Conclui-se que os acadêmicos possuem um bom conhecimento em relação à atuação do fisioterapeuta na UTI, porém condutas importantes atribuídas ao profissional tiveram baixos percentuais de reconhecimento, demonstrando que ainda há necessidade de uma maior integração entre os cursos.

Palavras-Chave: Conhecimento, Fisioterapeuta, Unidade de Terapia Intensiva.

\begin{abstract}
The Intensive Care Unit (ICU) is defined as a critical area for the hospitalization of critically ill patients, who requireongoing specialized professional attention, specific materials and technologiesnecessary for diagnosis, monitoring and therapy. To provide adequate assistance, a legally qualified multiprofessional team must be designated, and the duties and responsibilities of all professionals working in the unit must be formally assigned, described and disclosed to professionals and academics. The aim of this study was to identify the level of knowledge of health students from a higher education institution in Goiás about the role of the physiotherapist in the ICU multiprofessional team. This is a transverse and descriptive quantitative study, with 73 academics of Nursing, Medicine and Nutrition. It was used as a survey instrument developed by the researchers specifically for this study. Statistical analysis was performed using GraphPad Prism software version 5.0. Was analyzed significance of the questionnaire in the study with the student $t$ test paired parametric, was defined to study a significance level of $p$ $<0.05$. It was found that only the medical school's academic had significant results, about the knowledge of Physiotherapist conduct however the outcome of other courses was satisfactory considering the batting average above $60 \%$. It was considered very important the presence of the physiotherapist
\end{abstract}


in the multidisciplinary ICU team. It was evident that the students have a good knowledge about the physical therapist's role in ICU, but important conduits had little recognition demonstrating that there is still room for further integration between courses.

Keywords: Knowledge, Physiotherapist, Intensive Care Unit.

\section{Introdução}

A Unidade de Terapia Intensiva (UTI) é um espaço dinâmico, destinado a oferecer suporte de vida a pacientes graves, contando com aparelhagem de tecnologia avançada que pode aumentar assim a expectativa de vida do paciente. É fundamental a monitorização contínua dos sinais e sintomas clínicos que o paciente apresenta durante sua permanência na UTI, a fim de minimizar possíveis complicações ${ }^{1-3}$.

Por ser um ambiente dinâmico e de contínuas inspeções são necessários vários cuidados ao paciente crítico em diferentes competências, daí a necessidade de uma equipe multidisciplinar para a assistência contínua do paciente, na qual se insere o fisioterapeuta ${ }^{1-4}$.

A Fisioterapia integra parte das Ciências da Saúde que visa buscar, através de técnicas e recursos, a preservação e recuperação dos sistemas do corpo humano direcionando sua atuação para a independência funcional do paciente, melhorando sua qualidade de vida. Ao final da década de 70 o fisioterapeuta começou a integrar a equipe multiprofissional das UTI's, complementando a assistência e cuidados intensivos fundamentais ${ }^{4,5}$.

É clara a necessidade e a importância do fisioterapeuta em relação à assistência aos pacientes críticos. Uma atuação do fisioterapeuta na UTI consiste no auxílio do suporte ventilatório, onde o mesmo é capacitado e detentor de conhecimentos específicos, podendo atuar em todas as etapas, e que por sua vez faz parte do plano terapêutico que assiste o paciente, visando à melhora de seu quadro clínico. De acordo com a Resolução da Diretoria Colegiada (RDC) da ANVISA No 7/2010, a Unidade de Terapia Intensiva deve ter no mínimo 01 (um) fisioterapeuta para cada dez leitos em todos os turnos ${ }^{6}$.

Como atuação específica na assistência ao paciente grave, o fisioterapeuta possui uma gama de técnicas e recursos que auxiliam os cuidados ao paciente crítico, e que juntamente com os recursos da equipe multidisciplinar com ações organizadas e dinâmicas na UTI, irão favorecer para a melhora significativa deste paciente ${ }^{7,8}$.

Diante do exposto, percebe-se a importância da equipe multidisciplinar na UTI, porém não menos importante é a comunicação, entendimento e objetivos em comum entre os seus membros. Devido à complexidade da assistência ao paciente internado na UTI é essencial que os membros da equipe multidisciplinar saibam executar suas funções em conjunto ${ }^{8,9}$.
Através da troca de informações interdisciplinares será definido o melhor tratamento para o paciente, e a assistência em conjunto poderá minimizar riscos e erros trazendo benefícios para sua recuperação $0^{8,9}$.

A identificação do conhecimento que os acadêmicos dos cursos de Enfermagem, Medicina e Nutrição da Pontifícia Universidade Católica de Goiás (PUC Goiás) possuem sobre a atuação do fisioterapeuta, especificamente em UTI, tem sua relevância a medida que avalia a existência da interdisciplinaridade entre os cursos da instituição de ensino superior, formadora de profissionais que compõem a equipe multiprofissional, permitindo a identificação de possíveis lacunas na formação dos mesmos no que diz respeito à atuação profissional, com vista a diminuir o isolamento entre as áreas e incentivando a vivência mais ampla e harmoniosa. Esta pesquisa também se torna relevante devido à escassez de pesquisas com esta temática.

Este estudo teve como objetivo identificar o nível de conhecimento dos acadêmicos dos cursos de Enfermagem, Medicina e Nutrição da PUC Goiás sobre a atuação do fisioterapeuta em Unidades de Terapia Intensiva.

\section{Métodos}

Esta pesquisa caracteriza-se como quantitativa do tipo transversal e descritiva, foi desenvolvida no período de Maio a Junho de 2014, após aprovação do projeto no Comitê de Ética e Pesquisa da PUC Goiás (CAAE 30383114.2.0000.0037).

A amostra foi composta por 73 estudantes, sendo 22 acadêmicos de Enfermagem, de um total de 30 alunos, perfazendo um percentual de 73,33\%; no curso de Medicina houve a participação de 28 alunos, de um total de 39 , resultando em $71,79 \%$ e dos acadêmicos de Nutrição, 23 alunos, de um total de 58 , participaram da pesquisa $39,65 \%$ da população.

Para a coleta de dados foi utilizado como instrumento um questionário elaborado pelas pesquisadoras especificamente para a presente pesquisa estudo, tendo como referência o estudo de Braz, Martins e Vieira Júnior14. O questionário foi composto pela identificação pessoal do acadêmico (curso, período, idade e sexo) e por questões sobre a atuação do fisioterapeuta na UTI. As questões tinham com objetivo de identificar o conhecimento que os acadêmicos tinham sobre as condutas atribuídas ao Fisioterapeuta em uma UTI, com quatorze alternativas possíveis a serem assinaladas sem limite 
máximo. Na Tabela 1 estão os itens descritos no questionário, e suas respostas corretas.

Tabela 1: Atribuições do Fisioterapeuta na UTI - Respostas corretas de cada item.

\begin{tabular}{|c|c|}
\hline Condutas do fisioterapeuta na UTI & Respostas \\
\hline 01 - Posicionamento funcional do paciente no leito & Sim e compartilhada \\
\hline 02 - Realização de traqueostomia & Não realiza \\
\hline 03 - Manuseio de ventilação mecânica & Sim e compartilhada \\
\hline 04 - Mobilização e exercícios físicos & $\begin{array}{l}\text { Sim somente o } \\
\text { fisioterapeuta }\end{array}$ \\
\hline 05 - Administração de medicamentos & Não realiza \\
\hline 06 - Estimulação elétrica neuromuscular & Sim e compartilhada \\
\hline 07 - Prescrição de aerossolterapia & Não realiza \\
\hline 08 - Aspiração traqueal & Sim e compartilhada \\
\hline 09 - Terapias de higiene brônquica & $\begin{array}{l}\text { Sim somente o } \\
\text { fisioterapeuta }\end{array}$ \\
\hline $\begin{array}{l}10 \text { - Mensuração da força muscular dos músculos } \\
\text { respiratórios }\end{array}$ & $\begin{array}{l}\text { Sim somente o } \\
\text { fisioterapeuta }\end{array}$ \\
\hline $\begin{array}{l}11 \text { - Desmame e extubação do paciente em } \\
\text { ventilação mecânica }\end{array}$ & Sim e compartilhada \\
\hline 12 - Prescrição de medicamentos & Não realiza \\
\hline 13 - Treinamento muscular respiratório & $\begin{array}{l}\text { Sim somente o } \\
\text { fisioterapeuta }\end{array}$ \\
\hline 14 - Terapias de expansão pulmonar & $\begin{array}{l}\text { Sim somente o } \\
\text { fisioterapeuta }\end{array}$ \\
\hline
\end{tabular}

Foi utilizado o programa Microsoft Office Excel versão 2010 para tabulação e computação dos dados. Definiu-se para o estudo um nível de significância de $p<0,05$, a análise estatística foi efetuada utilizando o software GraphPad Prism versão 5.0 onde foi analisada a significância do questionário aplicado no estudo, sendo realizado a estatística descritiva com o cálculo de medidas de tendência central para as variáveis contínuas, como média, mediana e desvio padrão, e cálculo das frequências absoluta e relativa percentual para as variáveis descontínuas. Em seguida, aplicou-se um teste de normalidade para distinguir as distribuições paramétricas e não paramétricas, com o intuito de comparar os resultados do questionário estratificados pelas variáveis sociodemográficas. Para as distribuições paramétricas foi utilizado o teste t de Student.

\section{Resultados}

A pesquisa foi realizada na PUC Goiás com os acadêmicos dos cursos de Enfermagem, Medicina e Nutrição, que estavam cursando disciplinas de terapia intensiva no referido semestre. Para participarem do estudo os acadêmicos deveriam ser maiores de 18 anos, de ambos os sexos e ter aceitado assinar oTermo de Consentimento Livre e Esclarecido (TCLE).

Foi observado que $61 \%$ dos acadêmicos do curso de Medicina eram do sexo feminino e $39 \%$ do sexo masculino. Já os acadêmicos da Enfermagem $95 \%$ eram do sexo feminino e 5\% do masculino, e na Nutrição $96 \%$ eram do sexo feminino e 4\% do sexo masculino.

Em relação ao questionário aplicado para avaliar o conhecimento que os acadêmicos têm sobre a atuação do fisioterapeuta na UTI, na primeira questão os itens 4, 9, 10, 13 e 14 são considerados respostas adequadas e são condutas exclusivas do fisioterapeuta. Os itens 1, 3, 6, 8 e 11 são respostas adequadas também, porém referem-se a condutas que são compartilhadas com outros profissionais. Já os itens $2,5,7$ e 12 são considerados como repostas incorretas, pois são condutas não atribuídas ao fisioterapeuta. $O$ percentual de acertos dos acadêmicos dos três cursos são mostrados na Figura 1.

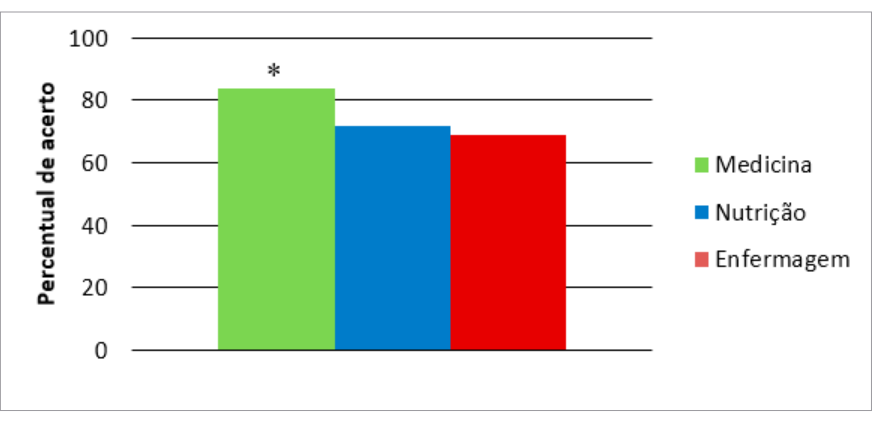

Figura 1: Percentual de acertos dos participantes do estudo em relação ás condutas exclusivas ou não do fisioterapeuta em UTI. ${ }^{*} p<0,05$. Figura 1. Percentual de acertos dos participantes do estudo em relação ás condutas exclusivas ou não do fisioterapeuta em UTI. ${ }^{*} p<0,05$.

Na tabela 2 são apresentadas as porcentagens dos itens que foram assinalados e não assinalados pelos participantes de cada curso no teste de conhecimento.

Tabela 2 - Respostas dos participantes do estudo sobre os itens que avaliam a conduta do fisioterapeuta na UTI.

\begin{tabular}{lcccccc}
\hline Item & $\begin{array}{c}\text { Medicina } \\
(\mathbf{n = 2 8})\end{array}$ & $\begin{array}{c}\text { Enfermagem } \\
(\mathbf{n = 2 2 )}\end{array}$ & $\begin{array}{c}\text { Nutrição } \\
(\boldsymbol{n}=\mathbf{2 3})\end{array}$ \\
\hline & $\operatorname{Sim}$ & Não & Sim & Não & Sim & Não \\
$\begin{array}{l}01 \text { - Posicionamento funcio- } \\
\text { nal do paciente no leito. }\end{array}$ & $75 \%$ & $25 \%$ & $41 \%$ & $59 \%$ & $91 \%$ & $9 \%$ \\
\end{tabular}




\begin{tabular}{|c|c|c|c|c|c|c|}
\hline $\begin{array}{l}02 \text { - Realização de } \\
\text { traqueostomia }\end{array}$ & $0 \%$ & $100 \%$ & $0 \%$ & $100 \%$ & $8 \%$ & $92 \%$ \\
\hline $\begin{array}{l}03 \text { - Manuseio de } \\
\text { ventilação mecânica }\end{array}$ & $61 \%$ & $39 \%$ & $86 \%$ & $14 \%$ & $35 \%$ & $65 \%$ \\
\hline $\begin{array}{l}04 \text { - Mobilização e } \\
\text { exercícios físicos }\end{array}$ & $100 \%$ & $0 \%$ & $100 \%$ & $0 \%$ & $91 \%$ & $9 \%$ \\
\hline $\begin{array}{l}05 \text { - Administração de } \\
\text { medicamentos }\end{array}$ & $4 \%$ & $96 \%$ & $0 \%$ & $100 \%$ & $0 \%$ & $100 \%$ \\
\hline $\begin{array}{l}06 \text { - Estimulação } \\
\text { elétrica neuromuscular }\end{array}$ & $64 \%$ & $36 \%$ & $50 \%$ & $50 \%$ & $52 \%$ & $48 \%$ \\
\hline $\begin{array}{l}07 \text { - Prescrição de } \\
\text { aerossolterapia }\end{array}$ & $4 \%$ & $96 \%$ & $14 \%$ & $86 \%$ & $39 \%$ & $61 \%$ \\
\hline 08 - Aspiração traqueal & $82 \%$ & $18 \%$ & $27 \%$ & $73 \%$ & $26 \%$ & $74 \%$ \\
\hline $\begin{array}{l}09 \text { - Manobras de higiene } \\
\text { brônquica }\end{array}$ & $82 \%$ & $18 \%$ & $18 \%$ & $82 \%$ & $52 \%$ & $48 \%$ \\
\hline $\begin{array}{l}10 \text { - Mensuração da força } \\
\text { muscular dos músculos } \\
\text { respiratórios }\end{array}$ & $96 \%$ & $4 \%$ & $91 \%$ & $9 \%$ & $87 \%$ & $13 \%$ \\
\hline $\begin{array}{l}11 \text { - Desmame e extubação } \\
\text { do paciente em ventilação } \\
\text { mecânica }\end{array}$ & $39 \%$ & $61 \%$ & $23 \%$ & $77 \%$ & $48 \%$ & $52 \%$ \\
\hline $\begin{array}{l}12 \text { - Prescrição de } \\
\text { medicamentos }\end{array}$ & $4 \%$ & $96 \%$ & $0 \%$ & $100 \%$ & $0 \%$ & $100 \%$ \\
\hline $\begin{array}{l}13 \text { - Treinamento } \\
\text { muscular respiratório }\end{array}$ & $100 \%$ & $0 \%$ & $100 \%$ & $0 \%$ & $96 \%$ & $4 \%$ \\
\hline $\begin{array}{l}14 \text { - Terapias de } \\
\text { expansão pulmonar }\end{array}$ & $93 \%$ & $7 \%$ & $100 \%$ & $0 \%$ & $78 \%$ & $22 \%$ \\
\hline
\end{tabular}

Verificou-se que somente os acadêmicos do curso de Medicina tiveram resultados significativos em relação aos demais cursos com uma média de acerto de 84\%, Enfermagem com $69 \%$ e Nutrição com $72 \%(p<0,05)$.

\section{Discussão}

A Unidade de Terapia Intensiva é um ambiente inconstante e de intensa carga de trabalho, pois a monitorização dos pacientes é contínua e a qualquer momento pode ocorrer situações de urgência. Diante disso percebe- se que o ambiente da UTI é estressante, tanto para pacientes quanto para os profissionais ${ }^{10}$.

Estudos têm evidenciado que a dificuldade para se trabalhar em equipe, má comunicação e falta de compromisso também são fatores estressantes para os membros da equipe. A segmentação da equipe com as atribuições de cada área do profissional geram consequências no processo do trabalho interdisciplinar ${ }^{10,11}$.

Segundo Araújo e Rocha' ${ }^{12}$, o trabalho em equipe tem como objetivo a obtenção de impactos sobre os diferentes fatores que interferem no processo saúde-doença. A ação interdisciplinar pressupõe a possibilidade da prática de um profissional se reconstruir na prática do outro, ambos sendo transformados para a intervenção na realidade em que estão inseridos.
Alves ${ }^{13}$ realizou um estudo sobre a importância da atuação do fisioterapeuta no ambiente hospitalar, fez uma revisão de 36 referências bibliográficas e concluiu que é indispensável a presença do fisioterapeuta durante 24 horas no ambiente hospitalar e salienta a necessidade de especialização na área por parte do profissional.

No estudo de Braz, Martins e Vieira Júnior ${ }^{14}$ foi analisado a atuação do fisioterapeuta em UTIs da cidade de Anápolis (GO), com a finalidade de observar a uniformidade ou não de atuações e a adequação quanto às habilidades e competências de um profissional dessa área. Eles concluíram que em relação ao conhecimento do fisioterapeuta, sobre a importância de sua inserção na UTI observou-se que os profissionais reafirmam a importância. Mas é difícil obter autonomia frente ao paciente crítico, por raramente haver credibilidade nessas profissões mais recentes da área da saúde, dificultando o aprimoramento de práticas e o reconhecimento da profissão.

Considerando os resultados da pesquisa verificou-se que a porcentagem de acerto foi acima de $60 \%$, demonstrando que os alunos possuem bom conhecimento sobre o papel do fisioterapeuta na UTI. Esses dados podem ser considerados satisfatórios à medida que a amostra foi composta por acadêmicos que estavam vivenciando pela primeira vez o ambiente de Terapia Intensiva.

Um dos fatores que pode ter contribuído para este resultado inclui a característica presente nos Projetos Pedagógicos dos cursos em questão, no que tange aos seus objetivos gerais da formação do profissional, que inclui o desenvolvimento de habilidades para atuar em equipes multiprofissionais, atuação essa que inclui o planejamento, implementação, coordenação, supervisão e o relacionamento interpessoal ético, em todos os contextos da atuação profissional ${ }^{15-16}$.

Conforme exposto na tabela 2 observa-se que os acadêmicos do curso de Medicina, possuem um maior conhecimento quanto às atribuições do fisioterapeuta na UTI, com uma diferença estatisticamente significativa em relação aos demais (Figura 1). Tal resultado pode ser atribuído às metodologias adotadas, como a problematização e a aprendizagem baseada em problemas (PBL- Problem - based learning) ${ }^{17}$.

De acordo com Berbel ${ }^{18}$ na metodologia da problematização, o grupo de alunos trabalham junto o tempo todo, com a supervisão de um professor. Em alguns momentos poderão distribuir tarefas, mas retornam sempre para o grupo, que vai construindo o conhecimento através das etapas do Arco. No PBL, inicia-se junto com o conhecimento e discussão do problema e retorna depois para a rediscussão no grupo tutorial, quando os estudos individuais já foram feitos. Professores especialistas podem ser consultados durante o estudo. 
Embora não significativo, os alunos do curso de Nutrição apresentaram resultados mais satisfatórios do que a Enfermagem, embora o segundo adote uma metodologia ativa de problematização, que também enfatiza o trabalho em grupo de forma prática e contínua.

Outro ponto a ser considerado, que pode ter contribuído para os resultados diz respeito à maturidade acadêmica e carga horária cursada pelos alunos até o momento de iniciação em ambiente de Terapia Intensiva. No curso de Medicina os alunos que estavam no Módulo XI cursaram até chegarem à prática da UTI o total de $6.660 \mathrm{~h}$, enquanto os alunos de Enfermagem do $6^{\circ}$ período cursaram $2.100 \mathrm{~h}$ e Nutrição do $8^{\circ}$ período $3.180 \mathrm{~h}$.

Nos resultados observou-se que condutas importantes do fisioterapeuta, tais como: posicionamento funcional do paciente no leito, manuseio de ventilação mecânica, aspiração traqueal, manobras de higienização brônquica, desmame e extubação, tiveram índices de erro maiores, demonstrando pouco reconhecimento por parte dos acadêmicos, o que representa a necessidade de uma maior integração entre as áreas durante o período de graduação.

É importante ressaltar que não foram encontrados estudos de temática semelhante publicados na literatura, para tanto, se mostra necessário pesquisas futuras nessa área de conhecimento.

\section{Conclusão}

Portanto os alunos concluintes dos cursos de Enfermagem, Medicina e Nutrição possuem nível satisfatório de conhecimento a respeito da atuação do fisioterapeuta em ambiente de terapia intensiva, embora tenham apresentado falta de conhecimento específico sobre condutas que são fundamentais para um bom desempenho de uma equipe multiprofissional. Desse modo percebe-se que ainda existe espaço para uma maior aproximação e integração entre os profissionais da saúde durante sua formação, pois durante sua atuação no mercado de trabalho é de extrema importância uma relação de confiança e respeito entre a equipe multidisciplinar, paciente e o fisioterapeuta.

Sendo assim, observa-se a necessidade de momentos que promovam a troca de informações e experiências, a fim de diminuir o isolamento entre as áreas e incentivar a vivência multiprofissional, otimizando a atuação interdisciplinar no campo de trabalho, o que sem dúvida terá um impacto positivo na vida destes futuros profissionais.

Por todos os aspectos analisados e devido à complexidade da pesquisa, conclui-se que devido à falta de literatura nessa área são necessários mais estudos para melhor compreender e estimular o trabalho multiprofissional, que deve ser iniciado desde a graduação.

\section{Referências}

1. Santuzzi CH, Scardua MJ, Reetz JB, Firme KS, Lira NO, Gonçalves WLS. Aspectos éticos e humanizados da Fisioterapia na UTI: uma revisão. Rev. Fisioterapia em Movimento. 2013;26(2):415-22. 2. Gosselink R, Clerckx B, Robbeets C, Vanhullebusch T, Vanpee G, Segers J. Physiotherapy in the Intensive Care Unit. Netherlands Journal of Critical Care. 2011;15 (2):66-75.

3. Denehy L, Berney S. Physiotherapy in the intensive care unit. Physical Therapy Reviews. 2006;11:49-56.

4. Nozawa E, Sarmento GJV, Vega JM, Costa D, Silva JEP, Feltrim MIZ. Perfil de fisioterapeutas brasileiros que atuam em unidades de terapia intensiva. Fisioterapia e Pesquisa. 2008;15(2):177-82. 5. França EET, Ferrari F, Fernandes P, Cavalcanti R, Duarte A, Martinez BP, et al. Fisioterapia em pacientes críticos adultos: recomendações do Departamento de Fisioterapia da Associação de Medicina Intensiva Brasileira. Rev. Bras. Ter. Intensiva. 2012;24(1):6-22.

6. Brasil. Agência Nacional de Vigilância Sanitária (ANVISA). Resolução nº 07 de 24 de fevereiro de 2010. Dispõe sobre os requisitos mínimos para funcionamento de Unidades de Terapia Intensiva e dá outras providencias. Diário Oficial da União 25 fev 2010; Seção 1.

7. Lopes FM, Brito ES. Humanização da assistência de fisioterapia: estudo com pacientes no período pós internação em unidade de terapia intensiva. Rev. Bras. Ter. Intensiva. 2009;21(3):283-91. 8. Cavalheiro LV, Andreoli PBA, Medeiros NS, Mendes TAB, Oliveira R, Cordeiro JJR. et al. Comunicação e acesso a informações na avaliação da qualidade de assistência multiprofissional a pacientes internados. Einstein. 2010; 8(3):303-7.

9. Ferreira CR, Varga RRC, Silva FR. Trabalho em equipe multiprofissional: a perspectiva dos residentes médicos em saúde da família. Ciência e Saúde Coletiva. 2009:14(supl. 1):1421-8.

10. Leite MA, Vila VSC. Dificuldades vivenciadas pela equipe multiprofissional na unidade de terapia intensiva. Rev Latino-am Enfermagem. 2005;13(2):145-50.

11. Silva CF, Souza DM, Pedreira LC, Santos MR, Faustino TN. Concepções da equipe multiprofissional sobre a implementação dos cuidados paliativos na unidade de terapia intensiva. Ciência e Saúde Coletiva. 2013;18(9).

12. Araújo MBS, Rocha PM. Trabalho em equipe: um desafio para a consolidação da estratégia de saúde da família. Ciência e Saúde Coletiva. 2007;12(2):455-64.

13. Nunes AA. A importância do fisioterapeuta no ambiente hospitalar. Ensaios e Ciência: Ciências Biológicas, Agrárias e da Saúde. 2012;16(6):173-84.

14. Braz PRP, Martins JOSOL, Vieira Júnior G. Atuação do fisioterapeuta nas unidades de terapia intensiva da cidade de Anápolis. Anuário de Produção Acadêmica Docente. 2009;3(4):119-29. 
15. Universidade Católica de Goiás. Departamento de Enfermagem e Fisioterapia. Projeto Pedagógico do curso de Enfermagem, 2006.

16. PUC Goiás. Departamento de Enfermagem Nutrição e Fisioterapia. Projeto Pedagógico do curso de Nutrição, 2009.

17. PUC Goiás. Departamento de Medicina. Projeto Político Pedagógico do curso de Medicina, 2011.

18. Berbel NAN. Metodologia da problematização: experiência com questões de ensino superior. Interface: Comunicação, Saúde, Educação. 1998;(2). 